\title{
For how much longer will we tolerate commercial screening?
}

Owing to an editorial error we misspelt the author's name in this Letter (BMJ 2012;344:e2729, doi:10.1136/bmj.e2729). It should have been David J Nicholl [not Nichol].

Cite this as: BMJ 2012;344:e2972

๑ BMJ Publishing Group Ltd 2012 